\title{
Vaping associated lung illness: a case series
}

\author{
Joanna Sesti ${ }^{1}$, Safiyya Quintiliani ${ }^{2}$, Christine Minerowicz ${ }^{3}$, Delyse Garg ${ }^{2}$, Shabiah Martin ${ }^{1}$, Karim Akl ${ }^{4}$, \\ Claudio Guerrieri ${ }^{5}$, Sabiha Hussain ${ }^{4}$, Christina Migliore ${ }^{2}$, Pratik Patel ${ }^{2}$, Marc Lindner $^{2}$, \\ Thiruvengadam Anandarangam ${ }^{2}$, Subroto Paul ${ }^{1}$
}

\begin{abstract}
${ }^{1}$ Department of Thoracic Surgery, RWJ Barnabas Health, West Orange, NJ, USA; ${ }^{2}$ Department of Pulmonology, Newark Beth Israel Medical Center, Newark, NJ, USA; ${ }^{3}$ Department of Pathology, Rutgers Robert Wood Johnson Medical School, New Brunswick, NJ, USA; ${ }^{4}$ Department of Pulmonology, Rutgers Robert Wood Johnson Medical School, New Brunswick, NJ, USA; ${ }^{5}$ Department of Pathology, Immunology and Molecular Pathology, Rutgers New Jersey Medical School, Newark, NJ, USA

Correspondence to: Joanna Sesti, MD. 101 Old Short Hills Road Ste. 302, West Orange, NJ 07052, USA. Email: Joanna.Sesti@rwjbh.org.
\end{abstract}

\begin{abstract}
Electronic cigarette (e-cigarette) use, also known as vaping, has gained popularity among young adults. It has been promoted as a safer alternative to conventional cigarette smoking. Recent reports, however, have linked e-cigarette use to lung dysfunction. We report a series of four patients who were recently treated within our hospital system for vaping associated lung illness (VALI). All four were young, healthy individuals with no significant past medical histories. Lung biopsies were performed on three of these patients and the results are reviewed in the article. All patients were treated with high dose steroids once cultures were finalized and no infectious etiology was detected. Recovery of lung function was seen after treatment in all cases.
\end{abstract}

Keywords: Case series; acute respiratory distress syndrome; vaping; e-cigarette

Received: 12 December 2019; Accepted: 26 December 2019; Published: 25 May 2020.

doi: $10.21037 /$ ccts.2019.12.12

View this article at: http://dx.doi.org/10.21037/ccts.2019.12.12

\section{Introduction}

Electronic cigarettes (e-cigarettes) are devices that heat liquid in prefilled cartridges which results in an aerosol or vapor that can be inhaled. Traditionally these cartridges contained nicotine and were promoted as a safer alternative to conventional cigarette smoking and a potential path for smoking cessation (1). Recently, vaping of the cannabinoid tetrahydrocannabinol (THC) has been seen especially among young adults. While cannabinoids are characterized as having anti-inflammatory properties, derivatives such as cannabidiol (CBD) have exhibited pro-inflammatory properties in the lungs of mouse models (2). Despite their rapid rise in popularity several reports have linked e-cigarette use to lung disease. We present a case series of four young, healthy patients with vaping exposure who were treated for respiratory failure along with a review of their corresponding pathologies. The cases are presented in accordance with the CARE guideline.

\section{Case presentation}

The first case is a 34-year-old male with history of childhood bronchitis who presented with progressive shortness of breath, fever, and malaise. He was employed as a restaurant manager. He had a remote history of polysubstance abuse including cigarette use for 10 years. One year prior to presentation he had transitioned to e-cigarettes. He used Juul nicotine products and reported 40-60 puffs per day.

On admission, he was afebrile and had an oxygen saturation of $80 \%$ on room air. He was started on high flow nasal cannula with improvement in his saturation levels. Computed tomography (CT) scan of his chest showed bilateral interstitial infiltrates with bibasilar consolidation (Figure 1). Echocardiogram did not reveal any valvular insufficiency and showed normal function. Blood, urine, and sputum cultures were negative for 


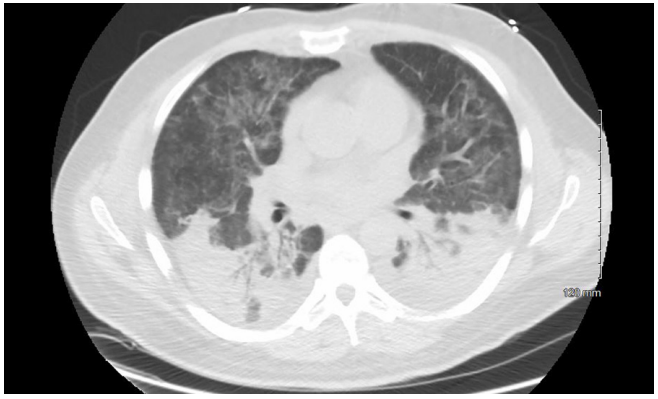

Figure 1 Non-contrast enhanced chest computer tomography in a 34-year-old male patient with vaping associated lung illness. Axial CT image of the chest shows ground-glass opacities in both lungs with dense bi-basilar consolidation.

microorganisms. A lung biopsy was performed which showed predominantly organizing pneumonia, with mild to focally moderate inflammation, focal collections of airspace foamy macrophages, and few areas with airspace fibrin (Figure $2 A, B, C$ ). Acute bronchiolitis and granulomas were not observed. The patient was started on methylprednisolone $40 \mathrm{mg}$ PO q 8 hours, and was discharged on hospital day 7 with maintenance therapy of $40 \mathrm{mg}$ prednisone twice a day and trimethoprim/sulfamethoxazole for Pneumocystis pneumonia prophylaxis. He was seen at two weeks from discharge and reported being back to baseline function.

The second case is a 24-year-old female with no significant past medical history who presented with progressive shortness of breath, fever, malaise, and emesis. She was employed as a waitress. She used Black Box marijuana e-cigarettes which she procured on the internet and reported 10-120 puffs per day. On admission, she was afebrile and profoundly hypoxic. The patient was intubated but continued to be profoundly hypoxic despite ventilator support. Due to worsening hypoxia, extracorporeal circulatory membrane oxygenation (ECMO) was instituted. A CT of the chest showed bilateral interstitial infiltrates with bibasilar consolidation (Figure 3). An echocardiogram did not reveal significant valvular insufficiency, showed normal left ventricular function, but the right ventricle was moderately to severely hypokinetic. Cultures of blood, urine, and sputum were sent and were negative. The presumed diagnosis was aspiration pneumonia related to a recent outpatient procedure which required conscious sedation; however, on hospital day 7 the patient's boyfriend, an e-cigarette user (see our patient \#3), was admitted with similar pulmonary symptoms and his lung biopsy showed findings somewhat similar to our first case. At that point,
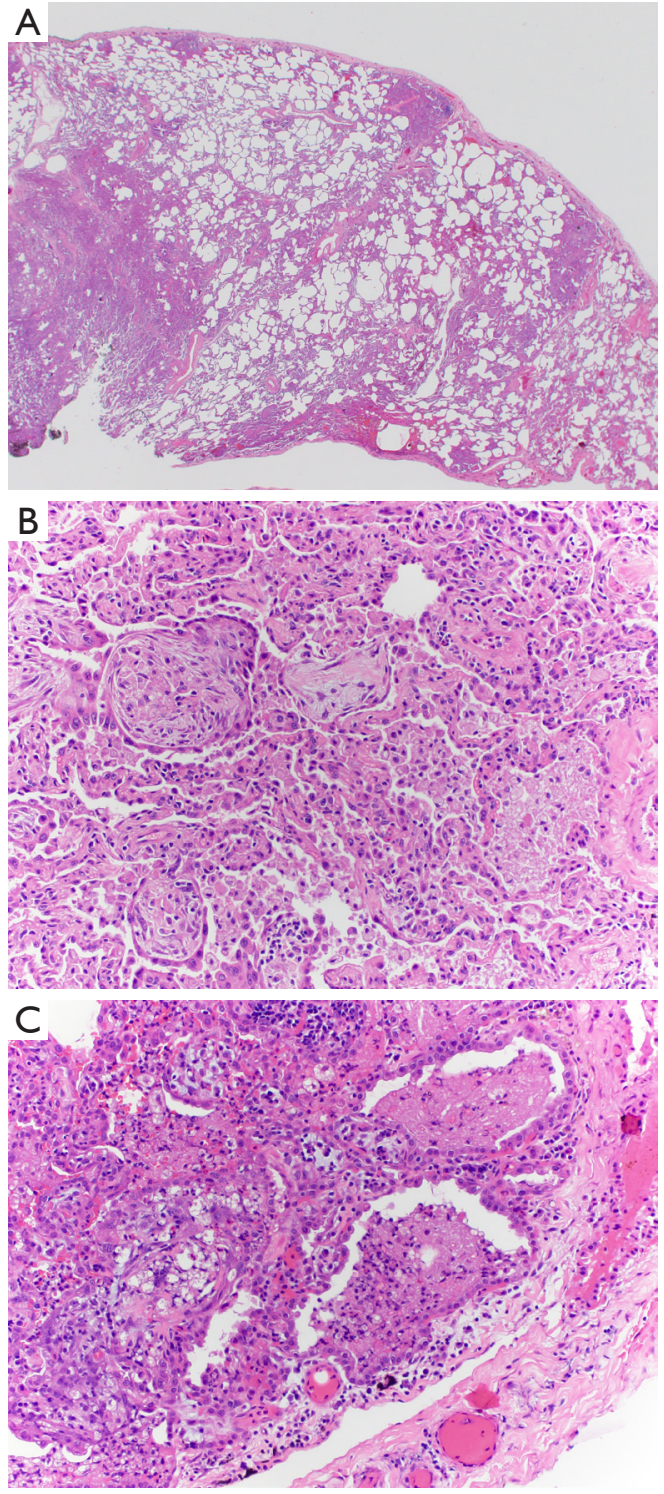

Figure 2 Lung biopsy of 34-year-old male with vaping associated lung illness (H\&E). (A) Low magnification image $(20 \times)$ shows patchy areas of lung injury alternating with unremarkable lung parenchyma. (B) High magnification image (200x) showing fibroblastic airspace plugs of organizing pneumonia, focal eosinophilic infiltrate and collection of vacuolated macrophages. (C) High magnification image (200x) of airspace fibrin with relative paucity of inflammatory cells.

she was started on $100 \mathrm{mg}$ of IV methylprednisolone daily. She was de-cannulated after 14 days of ECMO and eventually discharged on hospital day 23.

The third case is a 24-year-old male with a past medical history of gastroesophageal reflux disease and anxiety 


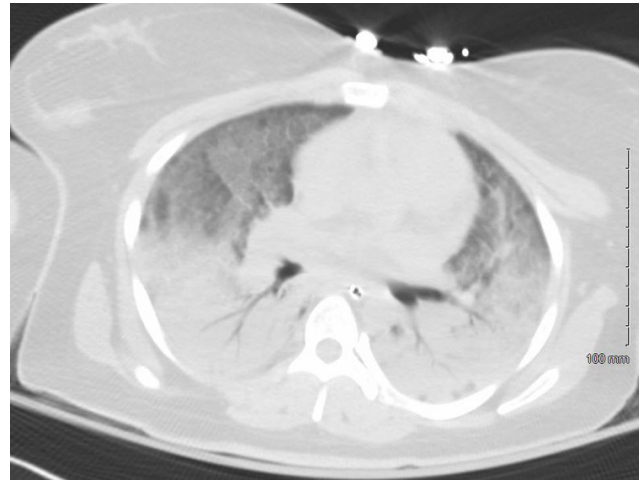

Figure 3 Non-contrast enhanced chest computer tomography in a 24-year-old female patient with vaping associated lung illness. Axial CT image of the chest shows ground-glass opacities in both lungs with dense bi-basilar consolidation.

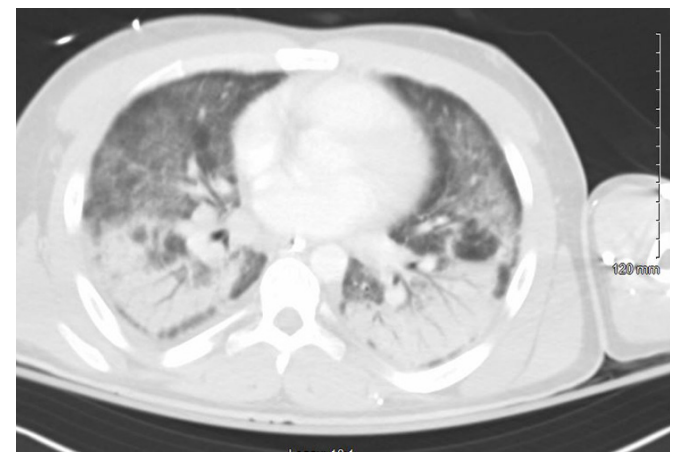

Figure 4 Non-contrast enhanced chest computer tomography in a 24-year-old male patient with vaping associated lung illness. Axial CT image of the chest shows ground-glass opacities in both lungs with dense bi-basilar consolidation.

who presented with progressive shortness of breath, fever, malaise, and emesis. He was employed as an arborist. He used Orion nicotine products as well as Black Box marijuana e-cigarettes, which he procured on the internet, and reported smoking throughout the day on a daily basis. On admission he was febrile and significantly hypoxic. The patient required intubation due to worsening hypoxia. CT of the chest showed bilateral interstitial infiltrates with bibasilar consolidation (Figure 4). Echocardiogram revealed trace tricuspid regurgitation, trace mitral regurgitation, and normal ventricular function. Cultures of blood, urine, and sputum were sent and eventually returned negative. A lung wedge biopsy showed an acute fibrinous and organizing pneumonia (AFOP) pattern of injury consisting of a patchy distribution of intra-alveolar balls of fibrinous
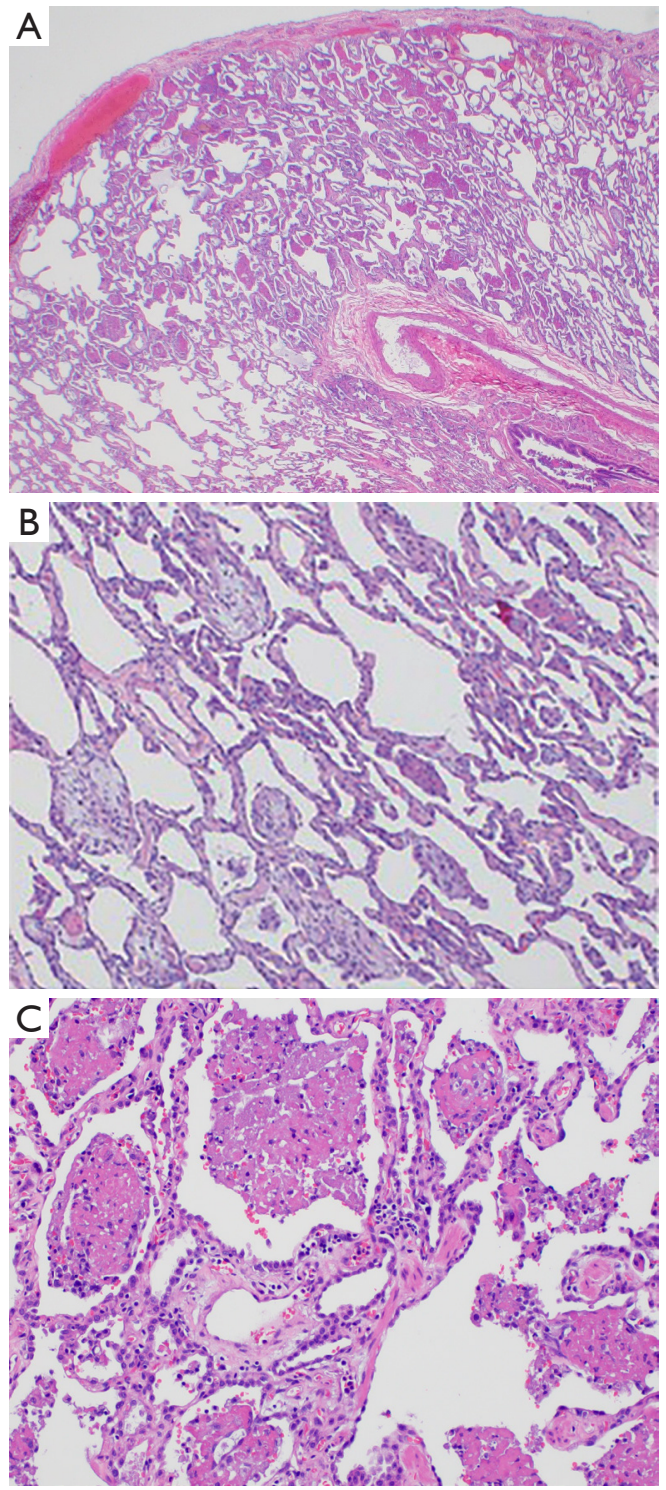

Figure 5 Lung biopsy of 24-year-old male with vaping associated lung illness (H\&E). (A) Low magnification image $(20 \times)$ shows patchy areas of airspace fibrin alternating with areas of unperturbed lung parenchyma. High magnification images showing organizing pneumonia (B) and airspace fibrin balls: $100 \times(\mathrm{C})$ with relative paucity of inflammatory cells: $200 \times$.

exudate associated with few neutrophils and only rare foamy macrophages, as well as scattered fibroblastic plugs within air spaces. (Figure $5 A, B, C$ ). Intraoperative pictures are shown in Figure 6. The patient was started on $125 \mathrm{mg}$ of IV methylprednisolone. He was discharged home on hospital day 9 with maintenance therapy of $60 \mathrm{mg}$ prednisone daily and trimethoprim/sulfamethoxazole for Pneumocystis 


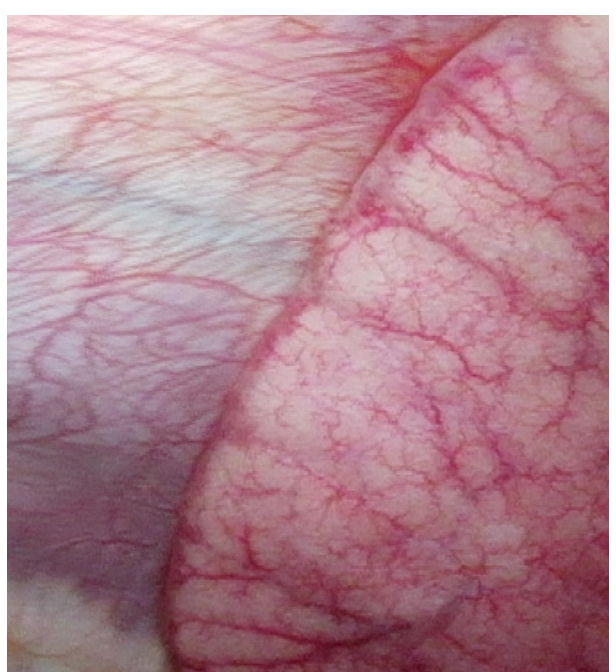

Figure 6 Intraoperative picture of lung in 24-year-old male with vaping associated lung illness. In vivo image of lung during right video-assisted thoracoscopic lung biopsy shows hypervascularity of the lung parenchyma and parietal pleura.

pneumonia prophylaxis. He was seen at two weeks from discharge and reported being back to baseline function.

The fourth case is a 20-year-old female college student with a past medical history of migraines who presented with worsening shortness of breath, cough, nausea and diarrhea for four days. She used e-cigarettes for two years with an increase from occasional use to daily in the month prior to presentation. She used a "Kind" pen with marijuana "smart card" refills, and reported 10-12 puffs per day. On her initial presentation to the emergency department she was diagnosed with community acquired pneumonia and sent home on Azithromycin. She returned 4 days later with fever, hypoxia and severe dyspnea. She was also noted to have leukocytosis. Her chest CT showed bilateral lower lobe infiltrates and she was treated with Vancomycin and Ceftriaxone. She became progressively more hypoxic and was intubated. Methylprednisolone $1,000 \mathrm{mg}$ daily was started, and her hypoxia improved. An infectious work-up was negative. She underwent lung biopsy which showed findings in keeping with AFOP (Figure $7 A, B$ ). She was discharged on day 8 on a prednisone taper.

All procedures performed in this study were in accordance with the ethical standards of the institutional and national research committees and with the Helsinki Declaration (as revised in 2013). Written consent was obtained from patients for publication of this case series and any accompanying images. A copy of the written consent is

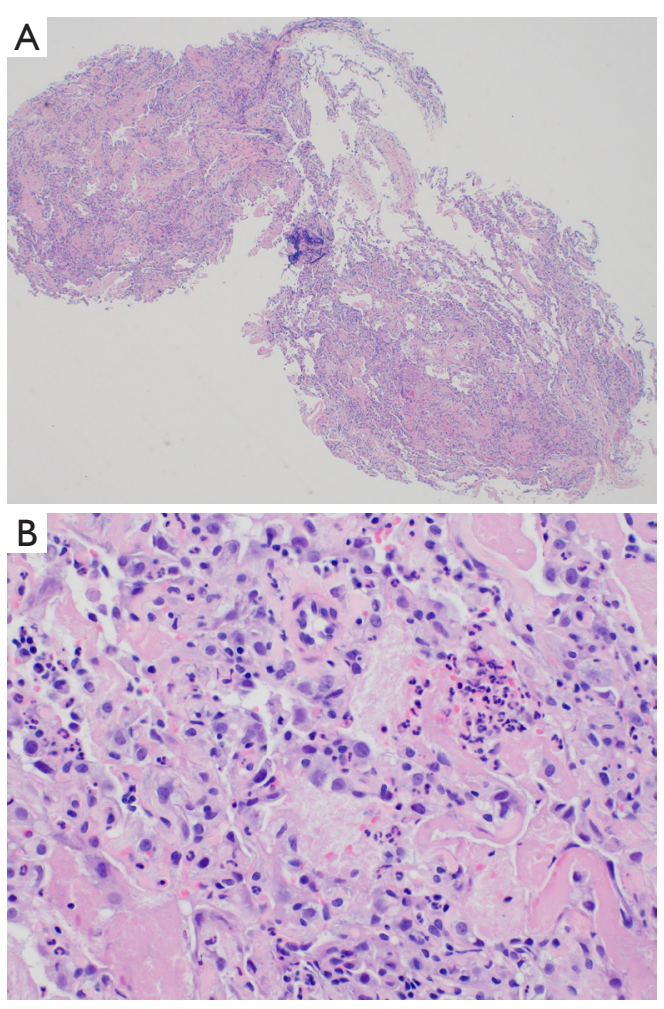

Figure 7 Lung biopsy of 20-year-old female with vaping associated lung illness (H\&E). (A) Low magnification image (40x) showing patchy airspace involvement. (B) High magnification image (400x) showing airspace fibrin and a small collection of neutrophils.

available for review by the editorial office of this journal.

\section{Discussion}

In the United States, e-cigarettes have become a multibillion dollar industry, with $\$ 2.5$ billion in sales in 2014 (3). The highest rates of use are reported in the 18-24 age group (4); however, the most rapid increase in use has been among adolescents. Between 2011 and 2015 the rate of e-cigarette use among high school students has risen 10fold (5). Part of the rapid expansion in use is the perceived notion that e-cigarettes are "safe." This designation however is not validated and necessarily true.

The lithium batteries that power these devices are susceptible to explosions and fires (6). Repeated heating cycles of the coil and wick components of the atomizer can lead to traces of metals such as nickel, chromium, and aluminum to leach into the e-liquid (7). Short term toxicity of inhaling fumes containing metal mixtures has been well 
appreciated in welders and is known as "metal fume fever" (8). E-liquids are composed of varying ratios of vegetable glycerin (VG), propylene glycol (PG), nicotine, and flavoring agents. Both VG and PG have been designated as "generally recognized as safe" (GRAS) by the FDA for oral intake; however, this designation does not apply to aerosolization. Long-term controlled studies looking at the effects of inhaling heated aerosolized VG or PG in humans are not available. Furthermore, flavoring components which may be an important motivator for e-cigarette use, especially in young consumers, historically has been linked to development of life-threatening respiratory failure (9).

Evidence of pulmonary toxicity from e-cigarette use in humans includes several studies that suggest an increased risk of chronic bronchitic symptoms, increased airflow resistance, decrease in exhaled nitric oxide suggesting increase in oxidative stress, and decreased cough sensitivity (10-12). Additionally, several case reports have recently drawn attention to the potential relationship between e-cigarette use and severe pulmonary disease. This has prompted a recommendation from the CDC (Centers for Disease Control and Prevention) to avoid use of e-cigarettes until more data is available (13). The largest case series was recently published from Wisconsin and Illinois (14). In this cohort, the majority of patients presented with respiratory, gastrointestinal, and constitutional symptoms. Chest imaging in all cases showed bilateral lung infiltrates. These characteristics are similar to the case reports in this series. The identification of lipid-laden macrophages that stain with oil red $\mathrm{O}$ in three BAL (bronchoalveolar) specimens from Utah has been suggested as a potential diagnostic tool (15).

Our case series highlights a clinical and radiographic presentation similar to the previously reported cases of vaping associated lung illness (VALI). Additionally, by expanding the spectrum of pathological findings that may be encountered in biopsy specimens of vaping-associated lung disease, we provide information which may aid in future identification of the underlying pathologic process resulting in lung injury. In our cases, the predominant histologic findings were organizing pneumonia (case 1) and AFOP (cases 3 and 4). It is possible that the biopsy results reflect acute hypersensitivity pneumonitis; however, the histologic features in such cases have not been well studied. Acute bronchiolitis has been described in acute hypersensitivity pneumonitis, which was absent in all our cases $(16,17)$. Case 1 showed focal collections of foamy macrophages as described above, but they were not felt to be extensive enough for a diagnosis of exogenous lipoid pneumonia, as was the reported primary pattern of injury in the recent outbreak in North Carolina (18). Aside from lipoid pneumonia, other reported manifestations of lung injury include organizing pneumonia, bronchiolitis, acute eosinophilic pneumonia, suspected acute hypersensitivity pneumonia, diffuse alveolar damage, respiratory bronchiolitis interstitial lung disease, and pneumonia with pleural effusion (19). The consistency in the pathologic finding of organizing pneumonia with varying degrees of airspace fibrin across our case series is intriguing, and clinicians and pathologists need to be aware that it is one of the possible manifestations of vaping-related lung injury. All four patients were treated with intravenous antibiotics and high dose corticosteroids. It remains unclear whether both of these treatments are necessary.

Further research is needed in identifying the exact agent at fault as well as the underlying disease process in VALI.

\section{Acknowledgments}

Funding: None.

\section{Footnote}

Conflicts of Interest: All authors have completed the ICMJE uniform disclosure form (available at https://ccts. amegroups.com/article/view/10.21037/ccts.2019.12.12/ coif). The authors have no conflicts of interest to declare.

Ethical Statement: The authors are accountable for all aspects of the work in ensuring that questions related to the accuracy or integrity of any part of the work are appropriately investigated and resolved. All procedures performed in this study were in accordance with the ethical standards of the institutional and national research committees and with the Helsinki Declaration (as revised in 2013). Written consent was obtained from patients for publication of this case series and any accompanying images. A copy of the written consent is available for review by the editorial office of this journal.

Open Access Statement: This is an Open Access article distributed in accordance with the Creative Commons Attribution-NonCommercial-NoDerivs 4.0 International License (CC BY-NC-ND 4.0), which permits the noncommercial replication and distribution of the article with the strict proviso that no changes or edits are made and the 
original work is properly cited (including links to both the formal publication through the relevant DOI and the license). See: https://creativecommons.org/licenses/by-nc-nd/4.0/.

\section{References}

1. Rom O, Pecorelli A, Valacchi G, et al. Are E-cigarettes a safe and good alternative to cigarette smoking? Ann N Y Acad Sci 2015;1340:65-74.

2. Karmaus PW, Wagner JG, Harkema JR, et al. Cannabidiol (CBD) enhances lipopolysaccharide (LPS)induced pulmonary inflammation in C57BL/6 mice. J Immunotoxicol 2013;10:321-8.

3. Herzog B GJ, Scott A. Equity Research: Tobacco - Nielsen C-store Data - E-cig \$ Sales Decline Moderates [Online]. In: Wells Fargo Securities: 2014. 25 Oct 2016. Available online: http://www.ecigarette-politics.com/files/4q14wells-fargo.pdf

4. CDC. Electronic Cigarette Use Among Adults: United States, 2014; Products - Data Briefs - Number 217 October 2015 [Online]. 12 Oct. 2016. Available online: http://www.cdc.gov/nchs/products/databriefs/db217.hrm

5. Singh T, Arrazola RA, Corey CG, et al. Tobacco Use Among Middle and High School Students--United States, 2011-2015. MMWR Morb Mortal Wkly Rep 2016;65:361-7.

6. US Fire Administration. Electronic Cigarette Fires and Explosions [Online]. FEMA 2014. Available online: https://www.usfa.fema.gov/downloads/pdf/publications/ electronic_cigarettes.pdf

7. Williams M VA, Bozhilov K, Lin S, Talbot P. Metal and silicate particles including nanoparticles are present in electronic cigarette cartomizer fluid and aerosol. PLoS One 2013;8.

8. Antonini JM, Taylor MD, Zimmer AT, et al. Pulmonary responses to welding fumes: role of metal constituents. J Toxicol Environ Health A 2004;67:233-49.

9. Kreiss K, Gomaa A, Kullman G, et al. Clinical

doi: $10.21037 /$ ccts.2019.12.12

Cite this article as: Sesti J, Quintiliani S, Minerowicz C, Garg D, Martin S, Akl K, Guerrieri C, Hussain S, Migliore C, Patel P, Lindner M, Anandarangam T, Paul S. Vaping associated lung illness: a case series. Curr Chall Thorac Surg 2020;2:20. bronchiolitis obliterans in workers at a microwave-popcorn plant. N Engl J Med 2002;347:330-8.

10. McConnell R, Barrington-Trimis JL, Wang K, et al. Electronic Cigarette Use and Respiratory Symptoms in Adolescents. Am J Respir Crit Care Med 2017;195:1043-9.

11. Vardavas CI, Anagnostopoulos N, Kougias M, et al. Shortterm pulmonary effects of using an electronic cigarette: impact on respiratory flow resistance, impedance, and exhaled nitric oxide. Chest 2012;141:1400-6.

12. Dicpinigaitis PV, Lee Chang A, Dicpinigaitis AJ, et al. Effect of e-Cigarette Use on Cough Reflex Sensitivity. Chest 2016;149:161-5.

13. Schier JG, Meiman JG, Layden J, et al. Severe Pulmonary Disease Associated with Electronic-Cigarette-Product Use - Interim Guidance. MMWR Morb Mortal Wkly Rep 2019;68:787-90.

14. Layden JE, Ghinai I, Pray I, et al. Pulmonary Illness Related to E-Cigarette Use in Illinois and Wisconsin Preliminary Report. N Engl J Med 2019. [Epub ahead of print].

15. Maddock SD, Cirulis MM, Callahan SJ, et al. Pulmonary Lipid-Laden Macrophages and Vaping. N Engl J Med 2019;381:1488-9.

16. Travis WD, Colby TV, Koss MN, et al. editors. NonNeoplastic Disorders of the Lower Respiratory Tract, Atlas of Nontumor Pathology First Series Fascicle 2. American Registry of Pathology and the Armed Forces Institute of Pathology. Washington, DC. 2002:101-102, 118-119.

17. Barrios, R. Hypersensitivity Pneumonitis (Extrinsic Allergic Alveolitis). In: Tomashefski JF Jr. editor. Dail and Hammar's Pulmonary Pathology. 3rd ed. Springer, 2008.

18. Davidson K, Brancato A, Heetderks P, et al. Outbreak of Electronic-Cigarette-Associated Acute Lipoid Pneumonia - North Carolina, July-August 2019. MMWR Morb Mortal Wkly Rep 2019;68:784-6.

19. Khan MS, Khateeb F, Akhtar J, et al. Organizing pneumonia related to electronic cigarette use: A case report and review of literature. Clin Respir J 2018;12:1295-9. 